\title{
Relation of birthweight to maternal plasma glucose and insulin concentrations during normal pregnancy
}

\author{
M.C.Breschi ${ }^{1}$, G.Seghieri ${ }^{1}$, G. Bartolomei ${ }^{1}$, A. Gironi ${ }^{1}$, S.Baldi ${ }^{2}$, E.Ferrannini ${ }^{2}$ \\ ${ }^{1}$ Diabetes Unit of the 2nd Medical Division, General Hospital, Pistoia, Italy \\ ${ }^{2}$ Metabolism Unit of the C.N.R. Institute of Clinical Physiology, University of Pisa, Pisa, Italy
}

\begin{abstract}
Summary. Maternal diabetes mellitus is complicated by fetal macrosomia and predisposes the offspring to diabetes, but recent evidence indicates that a low, not high, birthweight is associated with a higher incidence of Type 2 (non-insulin dependent) diabetes in adult life. To clarify the relationships between maternal glucose and insulin levels and birthweight, we measured oral glucose tolerance and neonatal weight in a large group $(n=529)$ of women during the 26 th week of pregnancy. Women with gestational diabetes $(n=17)$ had more familial diabetes, higher pre-pregnancy body weight, and tended to have large-for-gestational-age babies. In contrast, women with essential hypertension $(n=10)$ gave birth to significantly $(p<0.01)$ smaller babies. In the normal group (without gestational diabetes or hypertension, $n=503$ ), maternal body weight before pregnancy and at term, maternal height, week of delivery, gender of the newborn, and parity were all significant, independent predictors of birthweight, together explaining $23 \%$ of the variability of neonatal weight. In addition, both fasting $(p<0.006)$ and 2-h post-glucose $(p=0.03)$ maternal plasma glucose concentrations were positively associated with birthweight independent of the other physiological determi-
\end{abstract}

nants, accounting, however, for only $10 \%$ of the explained variability. In a subgroup of 134 normal mothers with prepregnancy body mass index of less than $25 \mathrm{~kg} \cdot \mathrm{m}^{-2}$, in whom plasma insulin measurements were available, the insulin area-under-curve was inversely related to birthweight $(p<0.02)$ after simultaneously adjusting for physiological factors and glucose area. When glucose and insulin measurements were combined in the $\mathrm{I} / \mathrm{G}$ ratio (ratio of insulin to glucose area), this was still inversely related to birthweight. Furthermore, maternal insulinaemia was directly related to blood pressure levels $(p<0.001)$ independently of body weight. We conclude that in normal pregnancy, whereas physiological factors account for most of the explainable variability of infant weight, the influence of the maternal metabolic milieu is dual, positive for glucose levels but negative for insulin concentrations. Maternal hyperinsulinaemia during pregnancy may be one trait linking low birthweight with predisposition to diabetes in adult life.

Key words: Birthweight, maternal hyperinsulinaemia, pregnancy, insulin resistance.
Fetal macrosomia is frequent in diabetic pregnancy [1]. According to Pedersen's [2] widely accepted hypothesis, fetal hyperinsulinaemia in response to maternal hyperglycaemia is the major cause of fetal macrosomia. During diabetic pregnancy, fetal macrosomia is related to maternal postprandial glucose control between the 29th and 32 nd week of gestation [3]. Furthermore, it has recently been shown that a subset of macrosomic infants born to non-diabetic mothers have higher insulin levels in cord blood [4]. Thus, it is possible that even subtle hyperglycaemia during pregnancy may lead to fetal hyperinsulinaemia and macrosomia, perhaps in conjunction with other factors (fetal beta-cell hypersensitivity, maternal amino acids, paternal glucose intolerance). In general, maternal glucose levels during normal pregnancy have been found to be positively associated with birthweight [5-8].

A series of retrospective studies [9-11] have demonstrated a strong link between low birthweight and impaired glucose tolerance (IGT), or Type 2 (non-insulindependent) diabetes, hypertension, and hyperlipidaemia - i.e., the components of the primary insulin resistance syndrome (or syndrome X [12]) - in adult life. These findings have suggested that the transmission of a genetic predisposition to glucose intolerance from the mother to the offspring may not be mediated by maternal glucose tolerance, and that Type 2 diabetes and hypertension have a common origin in sub-optimal development in utero [11]. In contrast, in a recent study low birthweight in first degree relatives of Type 2 diabetic subjects was associated 
Table 1. Anthropometric and biochemical characteristics of the study group ${ }^{\mathrm{a}}$

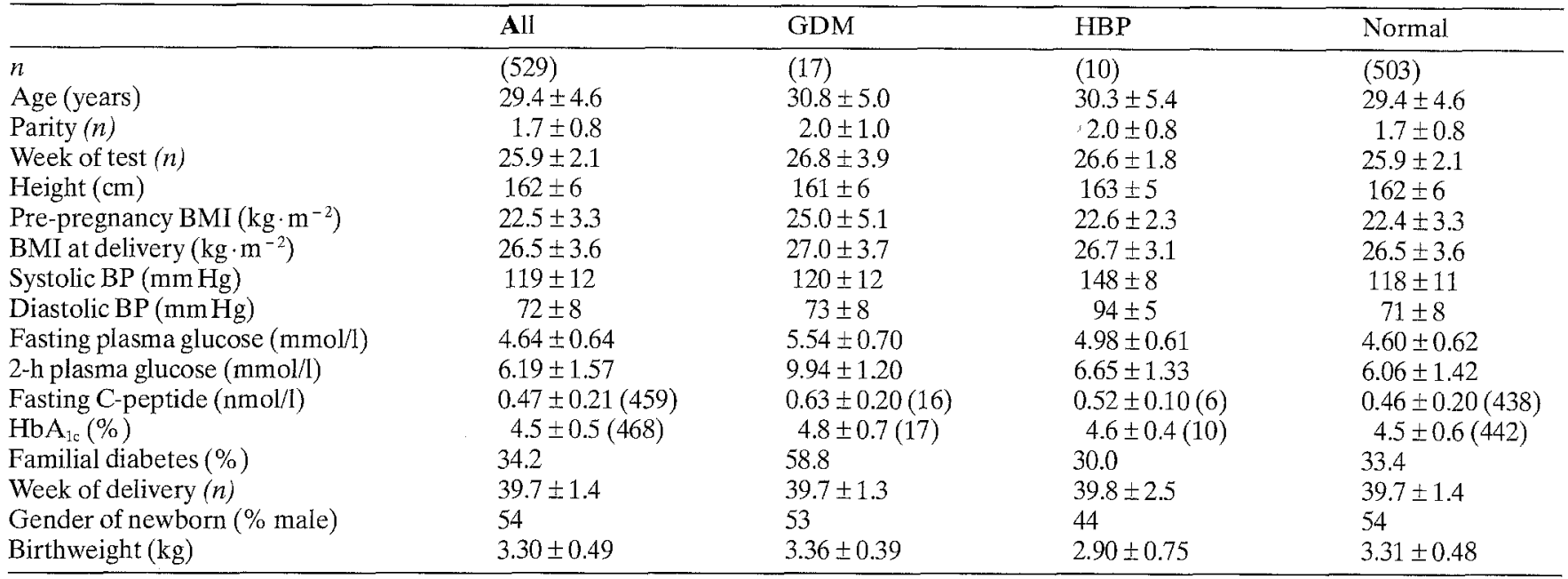

a Values are mean \pm SD. GDM, Gestational diabetes; HBP, hypertension; BMI, body mass index; number of observations in parentheses

Table 2. Multivariate analysis of birthweight in 503 normal women ${ }^{\text {a }}$

\begin{tabular}{lcccc}
\hline Independent variable & Regr. Coeff. & Std. Regr. Coeff. & \% Expl. Var. & $p$ value \\
\hline Intercept & -5.151 & 0 & - & $<0.0001$ \\
BMI at delivery & 0.046 & 0.353 & 4.6 & $<0.0001$ \\
Week of delivery & 0.101 & 0.302 & 9.1 & $<0.0001$ \\
Maternal height & 0.019 & 0.250 & 3.5 & $<0.0001$ \\
Gender of newborn & -0.166 & -0.174 & 0.0 & $<.0001$ \\
Pre-pregnancy BMI & -0.025 & -0.171 & 0.6 & 0.05 \\
Parity & 0.069 & 0.128 & 1.4 & 0.0016 \\
Fasting plasma glucose & 0.088 & 0.088 & 0.6 & 0.0055 \\
2-h plasma glucose & 0.029 & & 0.0343 \\
\hline
\end{tabular}

a Data from 503 women with normal glucose tolerance and blood pressure, tested (100-g OGTT) at the 26th week of pregnancy. The dependent variable is birthweight in $\mathrm{kg}$. Maternal height is in $\mathrm{cm}$, $\mathrm{BMI}$ in $\mathrm{kg} \cdot \mathrm{m}^{-2}$, sex is 0 for male, 1 for female, plasma glucose levels are in mmol per litre. The regression coefficients are given both in

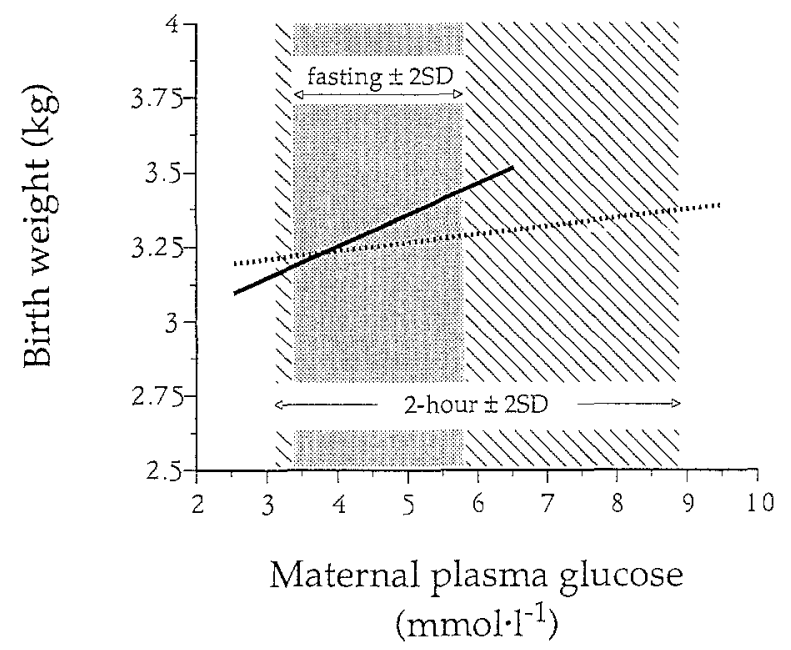

Fig. 1. Relationship between fasting (solid line, $p<0.006$ ) and 2-h post-glucose (dotted line, $p<0.04$ ) maternal plasma glucose concentrations during pregnancy (abscissae) and neonatal weight (ordinates) in 503 women with normal glucose tolerance and blood pressure. Data adjusted for all independent variables in Table 2, and calculated at the mean group values. The hatched areas denote the mean value $\pm 2 \mathrm{SD}$ of fasting and 2 -h plasma glucose levels in the whole group absolute value and in standardised form (i.e. independent of the measurement units), and are ranked according to the latter. The percent explained variance is the partial determination coefficient (squared partial correlation coefficient) multiplied by 100

with reduced beta-cell function at age 43 years, but did not predict the presence of IGT or diabetes [13].

These observations prompted us to re-examine the relationship between birthweight and glucose tolerance and insulin response during pregnancy.

\section{Subjects and methods}

The study group included 529 3rd-trimester pregnant women seen consecutively between 1988 and 1991 at a District Perinatal Clinic, and followed up until delivery. On interview, pre-pregnancy weight, parity, family history of diabetes, smoking habits, and antecedent macrosomia were recorded, and height, current weight, and arterial blood pressure (mean of last two of three measurements over 15 min using a sphygmomanometer with the subject in a sitting position) were measured. Women who had diabetes (Type 1 or Type 2) or other systemic diseases known before the present pregnancy were excluded from this report. On the following day after an overnight $(<12 \mathrm{~h}$ ) fast, all women received a $100 \mathrm{~g}$ oral glucose tolerance test (OGTT). Venous blood samples were drawn at baseline (fasting) and $2 \mathrm{~h}$ after glucose ingestion for plasma glucose measurement (by the glucose oxidase method). At delivery, week of delivery, body weight, and gender and weight of the newborn were recorded.

Gestational diabetes (GDM) was defined as a fasting venous blood glucose of $5.8 \mathrm{mmol} \cdot \mathrm{1}^{-1}$ or more and a 2 -h value of 
Table 3. Characteristics of lean and obese pregnant women ${ }^{3}$

\begin{tabular}{|c|c|c|c|}
\hline & Lean & Obese & $p$ value \\
\hline$n$ & 134 & 41 & \\
\hline Age (years) & $29.7 \pm 4.3$ & $29.2 \pm 5.2$ & NS \\
\hline Parity $(n)$ & $1.4 \pm 0.7$ & $1.5 \pm 0.7$ & NS \\
\hline Week of test $(n)$ & $26.7 \pm 2.7$ & $25.7 \pm 4.1$ & NS \\
\hline Height $(\mathrm{cm})$ & $163 \pm 5$ & $163 \pm 6$ & NS \\
\hline Pre-pregnancy body weight $(\mathrm{kg})$ & $57 \pm 6$ & $78 \pm 14$ & $<0.001$ \\
\hline Pre-pregnancy BMI $\left(\mathrm{kg} \cdot \mathrm{m}^{-2}\right)$ & $21.6 \pm 1.9$ & $29.6 \pm 5.2$ & $<0.001$ \\
\hline Weight at delivery $(\mathrm{kg})$ & $69 \pm 7$ & $88 \pm 14$ & $<0.001$ \\
\hline Weight increment (kg) & $11 \pm 3$ & $9 \pm 4$ & 0.01 \\
\hline Systolic BP (mm Hg) & $119 \pm 11$ & $124 \pm 13$ & $<0.04$ \\
\hline Diastolic BP (mm Hg) & $69 \pm 7$ & $75 \pm 9$ & $<0.001$ \\
\hline Mean BP (mmHg) & $85 \pm 7$ & $91 \pm 10$ & $<0.002$ \\
\hline $\mathrm{HbA}_{1 \mathrm{c}}(\%)$ & $4.4 \pm 0.4$ & $4.5 \pm 0.5$ & 0.06 \\
\hline Familial diabetes (\%) & 46.3 & 51.2 & NS \\
\hline Week of delivery $(n)$ & $39.7 \pm 1.3$ & $39.5 \pm 1.7$ & NS \\
\hline Gender of newborn (\% male) & 52 & 54 & NS \\
\hline Birthweight ( $\mathrm{kg}$ ) & $3.35 \pm 0.49$ & $3.46 \pm 0.55$ & NS \\
\hline
\end{tabular}

${ }^{a}$ Data of the 175 mothers in whom plasma insulin measurements were available. Values are mean \pm SD. BMI, Body mass index. ${ }^{b}$ By unpaired $t$ test or chi-square

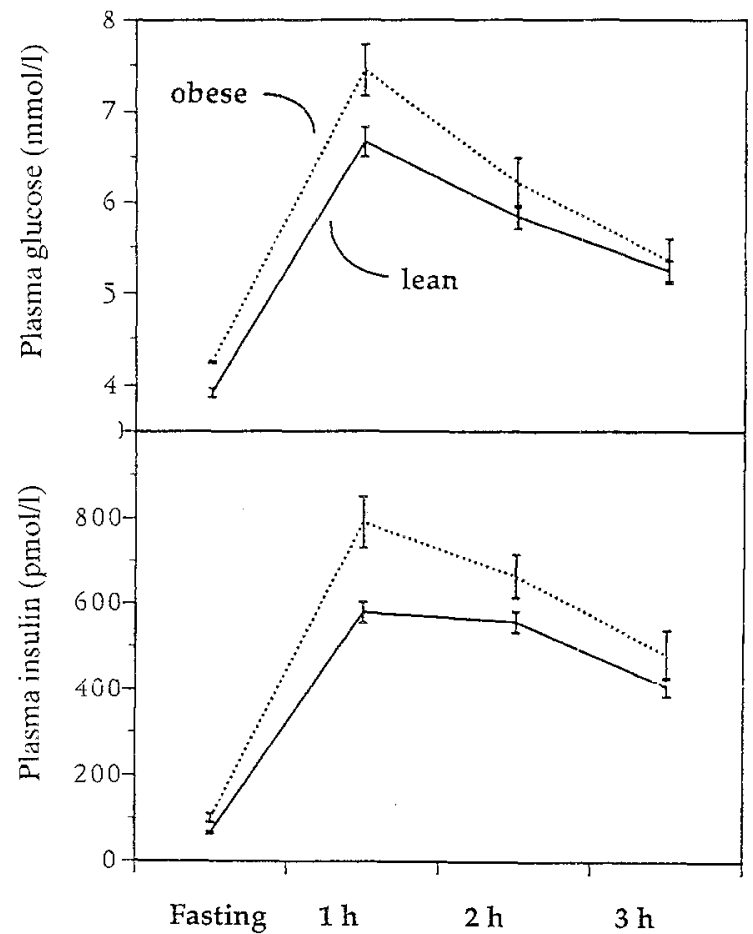

Fig. 2. Plasma glucose (top) and insulin (bottom) concentrations in the fasting state and following the ingestion of $100 \mathrm{~g}$ of glucose in 41 normal women with a pre-pregnancy $B M I \geq 25 \mathrm{~kg} \mathrm{~m}^{-2}$ (obese) and 134 normal women with a pre-pregnancy BMI $<25 \mathrm{~kg} \mathrm{~m}^{-2}$ (lean) at the 27 th week of pregnancy. Mean values are significantly $(p<0.05$ or less) higher at all time points in obese than in lean mothers

$9.2 \mathrm{mmol} \cdot \mathrm{l}^{-1}$ or more [14]. Hypertension was defined as a systolic blood pressure of $140 \mathrm{mmHg}$ or more and a diastolic value of $90 \mathrm{mmHg}$ or more [15]. Hypertension was diagnosed if repeated blood pressure measurements made by the same physician (M.C.B.) before the 20 th week of pregnancy met the above criteria. Since none of the hypertensive women had proteinuria over $0.3 \mathrm{~g}$ day $^{-1}$, a diagnosis of essential hypertension was considered likely.

In all women seen at the clinic after January 1991, additional blood samples were taken $1 \mathrm{~h}$ and $3 \mathrm{~h}$ after glucose ingestion, and plasma glucose and insulin concentrations were measured by radioimmunoassay (InsKit; Sorin Biomedica, Saluggia, Italy) on all samples. Data from 175 women without gestational diabetes or hypertension were available for analysis of insulin data.

\section{Statistical analysis}

Data are given as mean \pm SD. Body mass index (BMI) was calculated as the ratio of body weight in $\mathrm{kg}$ to the square of height in meters. A pre-pregnancy BMI of $25 \mathrm{~kg} \cdot \mathrm{m}^{2}$ was used as the cut-off to divide mothers into obese and lean. Mean blood pressure was calculated as the sum of the diastolic blood pressure and one third of the differential pressure. Areas under curve were computed by trapezoidal integration. Comparison of mean group values for continuous variables was performed with the use of Student's $t$-test; comparison of frequencies was carried out with the chi-square test. Insulin parameters showed a skewed distribution, and therefore were logarithmically transformed before inclusion into regression models. The simultaneous dependence of a continuous variable on several others was calculated by multiple linear regression.

\section{Results}

Seventeen women $(3.2 \%)$ had GDM on the basis of the $100-\mathrm{g}$ OGTT. Hypertension was present in another 10 women $(1.9 \%)$, one of whom also had GDM. The anthropometric and biochemical characteristics of the three groups (normal, GDM, and hypertensive) are given in Table 1. There were no significant differences in age, parity, week of delivery, BMI at delivery, or gender of newborn among the three groups. By contrast, the women with GDM tended to have a higher pre-pregnancy BMI $(t=1.89, p=0.06)$, significantly more diabetes in the family $\left(\chi^{2}=4.72, p=0.03\right)$, and higher fasting and 2-h blood glucose levels ( $p<0.0001$ for both) than normal women. The hypertensive women, on the other hand, in addition to higher levels of systolic and diastolic blood pressure ( $p<0.0001$ for both), tended to have higher blood glucose values at fasting $(t=1.91, p=0.06)$. In comparison with the normal group, birthweight was slightly but not significantly higher in the women with GDM, whereas it was sig- 

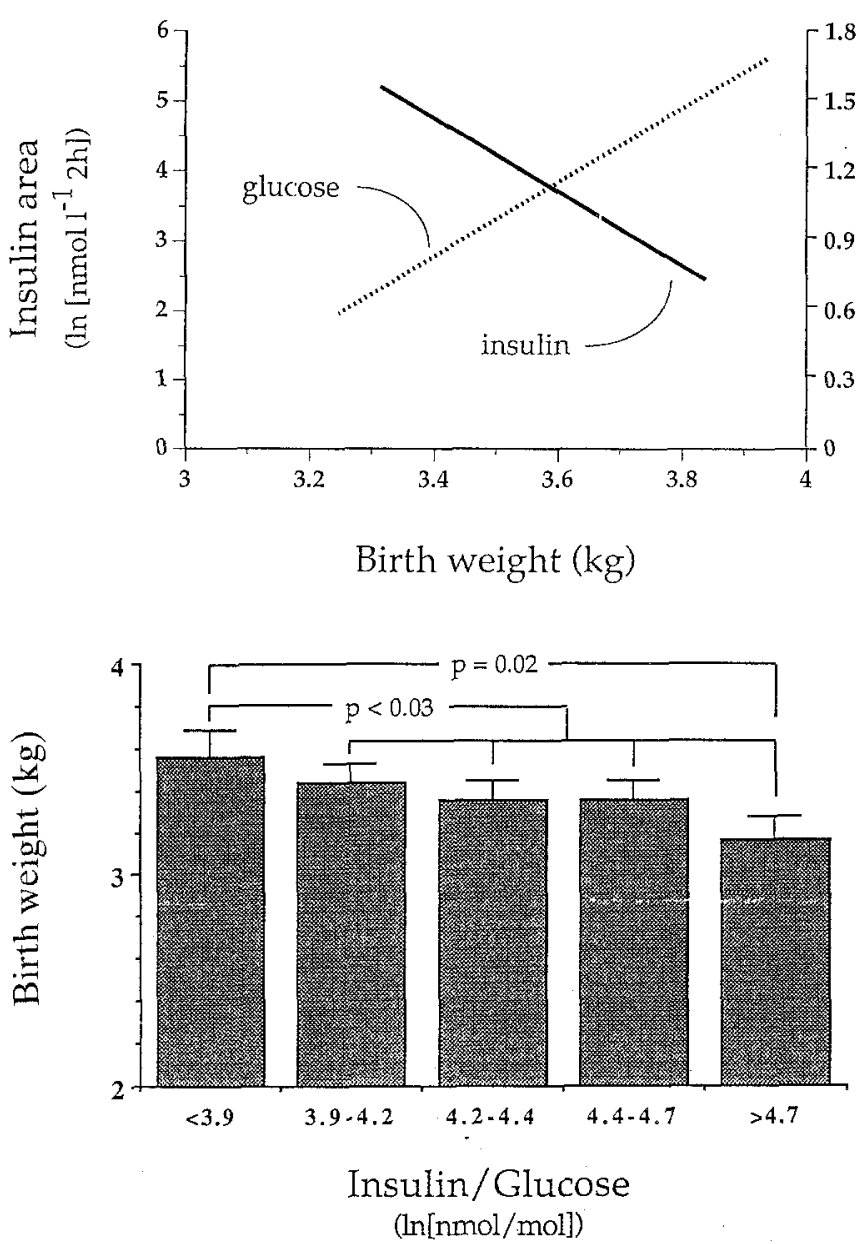

Fig. 4. Mean ( $\pm S E M)$ values for birthweight in quintiles of insulin/glucose ratio in 134 mothers (with normal glucose tolerance, blood pressure, and pre-pregnancy BMI) at the 27 th week of pregnancy. The $p$ values refer to $t$ tests between the upper and lower quintiles, or between the upper quintile and the mean of all the other values

nificantly lower in the small group of hypertensive women $(p<0.01)$.

In the whole group, birthweight was a normally distributed variable. In univariate analysis of the data of the normal women $(n=503)$, maternal height, BMI before pregnancy and at delivery, parity, week of delivery, fasting and 2-h plasma glucose concentrations, and male gender of the newborn were all significant positive correlates of birthweight, whereas maternal age, familial diabetes; systolic and diastolic blood pressure were not. Smoking was not significantly related to neonatal weight, presumably because only 63 of 503 women were smokers $(12.5 \%)$, and even these mothers smoked only 5 (median value) cigarettes per day. In a multiple regression equation using only the variables with significant univariate correlation coefficients, maternal height, BMI at baseline and delivery, parity, week of delivery, fasting and 2-h plasma glucose concentrations, and male gender of the newborn all remained significantly related to birthweight, and together explained $26 \%$ of the observed variance of this parameter (Table 2). Figure 1 shows the statistical dependence of neonatal weight on fasting and 2-h plasma glucose levels in non-diabetic, normotensive women after

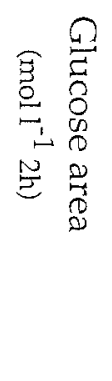

Fig. 3. Relationship between birthweight and the insulin and glucose area under curve in 134 mothers (with normal glucose tolerance, blood pressure, and pre-pregnancy BMI) at the 27 th week of pregnancy. The regressions were calculated from a multiple regression equation including week of delivery, maternal height, gender of newborn, glucose and insulin area by using the mean group values for the independent variables. The regression for insulin is adjusted for glucose and vice versa. The lines are drawn within the observed range of values for insulin and glucose area, respectively

adjusting for all the other significant correlates of the equation in Table 2. The slope of the regression line for fasting glucose is steeper than that for 2 -h glucose concentrations; it predicts $(p<0.006)$ an overall mean increment in birthweight of $0.66 \mathrm{~kg}$ over the observed range ( $\pm 2 \mathrm{SD}$ ) of fasting glucose levels (3.36 to $5.80 \mathrm{mmol}^{-1}$ ). The corresponding values for the observed range of post-glucose glycaemia ( 3.23 to $8.89 \mathrm{mmol} \cdot 1^{-1}$ ) is only $0.10 \mathrm{~kg}$ $(p<0.04)$.

The subsample $(n=175)$ of normal women in whom plasma insulin measurements were available was similar to the whole sample in all respects (data not shown). In this subgroup, birthweight was found to be inversely related to the insulin area (partial $r=0.13, p=0.05$ ) in a multiple regression model including maternal height, neonatal gender, week of delivery, and glucose area. To assess the influence of obesity, we subdivided this group of women into those who were overweight (BMI $\geq$ $\left.25 \mathrm{~kg} / \mathrm{m}^{2}\right)$ and those who were lean $\left(\mathrm{BMI}<25 \mathrm{~kg} / \mathrm{m}^{2}\right)$ before pregnancy. As shown in Figure 2, in the obese mothers - whose characteristics are compared with those of the lean group in Table 3-glucose tolerance was marginally worse than that of the lean women (glucose area $1.11 \pm 0.25 \mathrm{vs} 1.03 \pm 0.22 \mathrm{~mol} \cdot \mathrm{l}^{-1} \cdot 2 \mathrm{~h}, p<0.05$ ) in the face of a markedly heightened insulin response (insulin area $105 \pm 46 \mathrm{vs} 83 \pm 37 \mathrm{nmol} \cdot 1^{-1} \cdot 2 \mathrm{~h}, p<0.01$ ), i. e., they were insulin resistant (Fig.2). Also, the obese mothers gained significantly less weight than the lean mothers.

In the 134 non-diabetic, normotensive women with normal pre-pregnancy BMI, birthweight was still inversely $(p<0.02)$ associated with maternal insulinaemia after accounting for week of delivery, maternal height, and neonatal gender, and after simultaneously adjusting for maternal glycaemia (Fig. 3). The proportion of the total observed variance of neonatal weight that was explained by the measured variables was $29 \%$, similar to that $(26 \%)$ of the whole study group. Of this $29 \%$, about $3 \%$ each could be ascribed to the independent effect of maternal glucose and insulin levels.

When the insulin levels were corrected for the glucose concentrations by calculating the ratio of insulin to glucose area-under-curve ( $I / G$ ratio), birthweight in lean mothers was inversely related to the $\mathrm{I} / \mathrm{G}$ ratio both in univariate analysis $(r=0.20, p=0.02)$ and in multivariate analysis (partial $r=0.19, p<0.02$ ) after adjusting for 


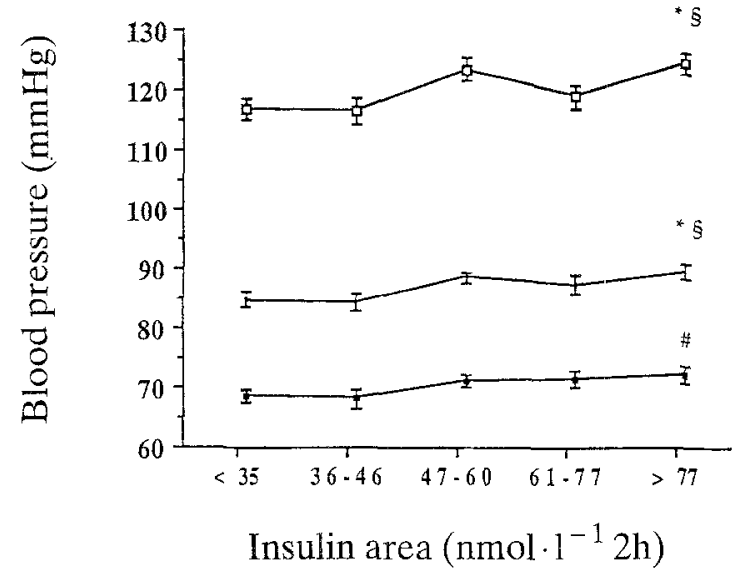

Fig.5. Systolic ( $\square$ ), diastolic ( $\mathbf{m}$ ), and mean ( - ) blood pressure by quintiles of insulin area in 134 mothers (with normal glucose tolerance, blood pressure, and pre-pregnancy BMI) at the 27 th week of pregnancy. " $p<0.03-0.02$ for the comparison (by $t$ test) of the upper quintile with the mean of all the other values. $\S p<0.01-$ 0.005 for the comparison between the upper and lower quintiles. \# $p=0.05$ for the comparison of the upper with the lower quintile

week of delivery, maternal height, and neonatal gender. This independent association remained negative and significant when the glucose area was separately added to the model, and when obese mothers were included in the analysis.

To analyse the characteristics of the mothers with a high $\mathrm{I} / \mathrm{G}$ ratio, the group of lean mothers $(n=134)$ was subdivided into quintiles of $\mathrm{I} / \mathrm{G}$ ratio. As shown in Figure 4 , women with the highest $\mathrm{I} / \mathrm{G}$ ratio had significantly smaller babies as compared to either the lowest I/G quintile or to all other quintiles together. However, there were no significant interquintile differences in age, BMI before pregnancy, during testing, or at term, week of delivery, or glucose area. Systolic $(121 \pm 8$ vs $116 \pm 9$ $\mathrm{mmHg}, p<0.04)$ and mean $(87 \pm 6$ vs $83 \pm 7 \mathrm{~mm} \mathrm{Hg}$, $p=0.06$ ) blood pressure levels were higher in the upper compared to the lower quintile. The relationship between blood pressure and insulin concentrations was examined by plotting the mean systolic, diastolic, and mean blood pressure values by quintiles of insulin area. As can be appreciated from Figure 5, there was a clear trend for all three blood pressure values to increase progressively across quintiles of insulin area. The same results were obtained when the incremental insulin area was used. Further analysis of this association was carried out by regression. Mean blood pressure was found to be significantly $(p=0.001)$ related to insulin area even after adjustment for BMI $(p=0.18)$ or maternal height $(p<0.01)$. Together with the latter, the insulin area explained $12 \%$ of the variability of mean blood pressure.

\section{Discussion}

The present study confirms that the presence of high blood pressure in the mother is associated with mildly impaired glucose tolerance (if still within the normal limits), and a lower birthweight $[16,17]$. Women with GDM, on the other hand, had higher pre-pregnancy weight and more familial diabetes, as expected; a trend towards higher neonatal weight was also present, but did not reach statistical significance in the small group of GDM women included in the present series.

In the non-diabetic, normotensive mothers, physiological factors - such as week of delivery, maternal height and BMI, parity, and gender of the newborn - were the most important determinants of neonatal weight, together accounting for over $90 \%$ of the explainable variance of birthweight in this group. Thus, in studies in which birthweight is correlated with a variety of outcomes - such as adult blood pressure [16,17], IGT [5] or ischaemic heart disease [18] - these physiological determinants of neonatal weight should be taken into adequate account. It is important to note, however, that approximately $75 \%$ of the total observed variance of birthweight remains unexplained.

We, like others [5-8], found that maternal blood glucose levels are directly related to neonatal weight independently of the other physiological factors. Thus, the known association between maternal diabetes and high birthweight extends into the physiological range of glucose tolerance. Indeed, the multiple regression equation derived in our healthy mothers predicts a mean neonatal weight of $3.37 \mathrm{~kg}$ in the GDM group, i. e., almost identical to that actually observed $(3.36 \mathrm{~kg})$, suggesting a continuous relationship between maternal glucose tolerance during pregnancy and neonatal weight. In contrast, the same equation predicts a mean birthweight of $3.31 \mathrm{~kg}$ in the hypertensive mothers, in whom the observed value was almost $0.5 \mathrm{~kg}$ lower. This implies that, although blood pressure was not itself an independent determinant of birthweight in the normal population, the presence of clinical hypertension in the mother overrides the impact of glucose tolerance, and is associated with a low birthweight even after adjustment for the other factors.

The first new finding of this study concerns maternal plasma insulin concentrations. In the subgroup of nondiabetic, normotensive mothers in whom insulin measurements were available, the insulin area was found to be inversely related to birthweight after adjustment for both the physiological factors and plasma glucose levels themselves. Since the insulin response to glucose is enhanced by obesity, maternal overweight might confound the relationship between plasma insulin levels and birthweight. In fact, mothers with a pre-pregnancy BMI $25 \mathrm{~kg} \cdot \mathrm{m}^{-2}$ or over were significantly hyperinsulinaemic compared to lean women despite similar or higher plasma glucose levels. However, the inverse association of insulin area with birthweight was equally present in the rigorously lean mothers, indicating that the link is not forced by obesity. In this group, again only less than one third of total birthweight variance could be explained by measured variables, and plasma glucose and insulin levels each accounted for only about $3 \%$ of it. It is important to emphasise that the opposing influences of plasma glucose and insulin concentrations on birthweight (a) are independent of one another, (b) are relatively weak, as they each explain a small part of birthweight variability, and (c) relate to the 26th week of pregnancy of strictly normal 
women, i.e. with normal body weight, blood pressure, and glucose tolerance.

When glucose and insulin levels were combined in the $\mathrm{I} / \mathrm{G}$ ratio, this was found to be significantly related to birthweight in an inverse fashion. Upon stratifying our women by quintiles of $\mathrm{I} / \mathrm{G}$ ratio, the glucose area was similar in all strata, indicating that higher insulin rather than lower glucose was responsible for the observed rank of $\mathrm{I} / \mathrm{G}$ ratios. Then, the fact that birthweight was significantly lower in the upper $\mathrm{I} / \mathrm{G}$ quintile in comparison with the other quintiles conceivably reflects an association between true maternal hyperinsulinaemia and neonatal weight.

The present findings are compatible with the classic sequence (predisposition to diabetes $\rightarrow$ maternal glucose intolerance $\longrightarrow$ large babies $\longrightarrow$ diabetes in the offspring), and extend the relation between maternal glycaemia and birthweight to normal pregnancy. On the other hand, the same findings may reconcile this sequence with the more recent observation, that a low birthweight and a lower weight at 1 year predict IGT during the sixth decade of life [9-11]. Maternal hyperinsulinaemia is associated with low birthweight, and might represent the trait through which the genetic pressure towards diabetes is routed to the offspring. In fact, it is now very well established that insulin resistance precedes and predicts diabetes independently of IGT [19]. To the extent that hyperinsulinaemia (or the $\mathrm{I} / \mathrm{G}$ ratio) reflect insulin sensitivity, our data suggest that maternal insulin resistance could channel its impact on future diabetes via low birthweight. Whether the hyperinsulinaemia/insulin resistance is itself a cause of early fetal malnutrition, or simply a marker for some underlying mechanism(s) remains to be tested.

The other new finding is the association of hyperinsulinaemia with higher blood pressure in our normal women during the third trimester of pregnancy. A relationship between plasma insulin concentrations (or insulin resistance) and blood pressure levels has been repeatedly reported both in normotensive and hypertensive groups [20-21]. During pregnancy, insulin resistance increases [22] whereas blood pressure levels fall [15] as part of a physiological adaptation. Nevertheless, we find here that insulin concentrations and blood pressure levels remain significantly associated with one another during normal pregnancy after adjusting for the common confounder, i. e., body size. This observation is in keeping with the hypothesis that a low birthweight predicts not only diabetes but also hypertension in adult life. Since blood pressure is a 'tracking' variable [23], normal mothers in the upper range of the blood pressure distribution would also develop more hyperinsulinaemia during pregnancy, give birth to relatively smaller babies, and thereby transfer the predisposition to hypertension to the offspring. Once again, the higher blood pressure could be a direct mechanism or a marker of mechanism(s) for reduced fetal growth $[16,17]$.

According to these considerations, mothers with overt diabetes transfer an increased risk of diabetes through larger babies because the positive effect of maternal hyperglycaemia on fetal growth (via fetal hyperinsu- linaemia) overrides the negative effect of any maternal hyperinsulinaemia on birthweight. In non-diabetic women, on the other hand, plasma glucose and insulin levels balance their reciprocal influence on birthweight. Since, however, insulin resistance is more common than hyperglycaemia in the general population, the prevalent association would be between low birthweight and adult syndrome $X$.

These hypotheses demand caution for at least two reasons. First, a direct demonstration of this dual pathway of maternal transfer of predisposition requires a prospective examination of mothers with known degrees of insulin resistance until enough disease cases have accumulated in their offspring. Second, in our database plasma glucose and insulin levels account for a small fraction of birthweight variability, a larger part being explained by physiological factors, and the largest part being unexplained. Possibly, early intrauterine changes in response to the maternal metabolic milieu are responsible for only some portion of diabetes incidence, perhaps explaining the excess diabetes associated with maternal compared to paternal Type 2 diabetes [24].

Acknowledgement. The authors wish to thank Mr. P. Belli for his skilled technical assistance.

\section{References}

1. Susa JB (1988) Effects of diabetes on fetal growth. In: Reece EA, Coustan DR (eds) Diabetes mellitus in pregnancy, principles and practice. Churchill Livingstone, New York, pp 105-122

2. Pedersen $\mathrm{J}$ (1954) Weight and length at birth of infants of diabetic mothers. Acta Endocrinol 16: 330-342

3. Combs CA, Gunderson E, Kitzmiller JL, Gavin LA, Main EK (1992) Relationship of fetal macrosomia to maternal postprandial glucose control during pregnancy. Diabetes Care 15: 12511257

4. Hoegsberg B, Gruppuso PA, Coustan DR (1993) Hyperinsulinemia in macrosomic infants of nondiabetic mothers. Diabetes Care 16:32-36

5. Tallarigo L, Giampietro O, Penno G, Miccoli R, Gregori G, Navalesi $R$ (1986) Relation of glucose tolerance to complications of pregnancy in nondiabetic women. N Engl J Med 315:989-992

6. Langhoff-Roos J, Wibell L, Gebre-Medhin M, Lindmark G (1988) Maternal glucose metabolism and infant birth weight: a study in healthy pregnant women. Diabetes Res 8: $165-170$

7. Farmer G, Russell G, Hamilton-Nicol DR et al. (1988) The influence of maternal glucose metabolism on fetal growth, development and morbidity in 917 singleton pregnancies in nondiabetic women. Diabetologia 31: 134-141

8. Klakhoff RK (1991) Impact of maternal fuels and nutritional state on fetal growth. Diabetes 40 [Suppl 2]: 61-65

9. Hales CN, Barker DJP, Clark PMS et al. (1991) Fetal and infant growth and impaired glucose tolerance at age 64. BMJ 303: $1019-1022$

10. Phipps K, Barker DJP, Hales CN, Fall CDH, Osmond C, Clark PMS (1993) Fetal growth and impaired glucose tolerance in men and women. Diabetologia 36: 225-228

11. Barker DJP, Hales CN, Fall CHD, Osmond C, Phipps K, Clark PMS (1993) Type 2 (non-insulin-dependent) diabetes mellitus, hypertension and hyperlipidaemia (syndrome X): relation to reduced fetal growth. Diabetologia 36: 62-67

12. Reaven GM (1988) Role of insulin resistance in human disease. Diabetes 37: 1595-1607

13. Cook JTE, Levy JC, Page RCL, Shaw JAG, Hattersley AT, Turner RC (1993) Association of low birthweight with $\beta$ cell function 
in the adult first degree relatives of non-insulin dependent diabetic subjects. BMJ 306: 302-306

14. Metzger BE and the Organizing Committee (1991) Summary and recommendations of the Third International WorkshopConference on Gestational Diabetes Mellitus. Diabetes 40 [Suppl 2]: 197-201

15. Gant NF, Worley RJ (1980) Hypertension in pregnancy: concepts and management. Appleton, New York, pp 1-8

16. Barker DJP, Osmond C, Golding J, Kuh D, Wadsworth MEJ (1989) Growth in utero, blood pressure in childhood and adult life, and mortality from cardiovascular disease. BMJ 298: 564-567

17. Barker DJP, Bull AR, Osmond C, Simmonds SJ (1990) Fetal and placental size and risk of hypertension in adult life. BMJ 301: 259-262

18. Barker DJP, Winter PD, Osmond C, Margetts B, Simmonds SJ (1989) Weight in infancy and death from ischaemic heart disease. Lancet II: $577-580$

19. DeFronzo RA, Bonadonna RC, Ferrannini E (1992) Pathogenesis of NIDDM: a precarious balance between insulin action and insulin secretion. In: Alberti KGMM, DeFronzo RA, Keen H, Zimmet P (eds) International textbook of diabetes mellitus. John Wiley \& Sons, Chichester, pp 569-634
20. Ferrannini E, Haffner SM, Stern MP (1990) Essential hypertension: an insulin resistant state. J Cardiovasc Pharmacol 15 [Suppl 5]: S18-S25

21. Ferrari P, Weidmann $P$ (1990) Insulin, insulin sensitivity and hypertension. J Hypertens 8: 491-500

22. Felig P (1977) Body fuel metabolism and diabetes mellitus in pregnancy. Med Clin North Am 61: 43-55

23. Florey C du V, Uppal S, Lowy C (1976) Relation between blood pressure, weight, and plasma sugar and serum insulin levels in schoolchildren aged 9-12 years in Westland, Holland. BMJ 1: 1368-1371

24. Vadheim CM, Rotter JI (1992) Genetics of diabetes mellitus. In: Alberti KGMM, DeFronzo RA, Keen H, Zimmet P (eds) International textbook of diabetes mellitus. John Wiley \& Sons, Chichester, pp 72-73

Received: 13 April 1993

and in revised form: 26 July 1993

Dr. E. Ferrannini

C.N.R. Institute of Clinical Physiology

Via Savi, 8

I-56100 Pisa

Italy 